\title{
Preferencias de los consumidores: Un elemento clave en el turismo alimentario
}

\author{
Consumer preferences: An essential element in food tourism
}

Dena Ma. Jesús Camarena Gómez*®

Camarena Gómez, D. M. J. (2017). Preferencias de los consumidores: Un elemento clave en el turismo alimentario. Investigación y Ciencia de la Universidad Autónoma de Aguascalientes, 25(71), 59-67.

RESUMEN

La comida siempre ha estado presente en las actividades turísticas por necesidad biológica, no obstante, en los últimos años se comenzó a considerar como un elemento clave en el desarrollo de este rubro, ha llegado incluso a representar uno más de los atractivos, con los aspectos culturales y naturales. El objetivo de la investigación fue identificar los alimentos tradicionales de una región turística del estado de Sonora, que pueden integrarse dentro del turismo alimentario a partir del conocimiento de las preferencias de los consumidores. Para ello se aplicó una encuesta dirigida a 262 potenciales visitantes mediante un experimento de elección, donde los atributos de precio, origen y tipo de alimento fueron los considerados. Para identificar las opciones preferidas se estimó un modelo logit condicional, en donde los tamales de carne procedentes de Ures, con un precio bajo, fueron el producto favorito.

\section{ABSTRACT}

Food always has been present in the tourist activities by biological necessity, however, in the last years, it

Palabras clave: turismo alimentario; experimento de elección; modelo logit; consumidor turístico; comida tradicional; tamales.

Keywords: food tourism; choice experiment; logit model; touristic consumer; traditional food; tamales.

\footnotetext{
Recibido: 26 de enero de 2017, aceptado: 13 de junio de 2017
}

* Departamento de Contabilidad, División de Ciencias Económicas y Administrativas, Universidad de Sonora. Blvd. Luis Encinas y Rosales $s / n, C$. P. 83000, Hermosillo, Sonora, México. Correo electrónico:

dena.camarena@eca.uson.mx

$凶$

Autor para correspondencia has started to be considered a principal element in the tourism development, even to represent one of the attractions of a touristic destination along with the cultural and natural aspects. In this context, the aim objective of this research was to identify the traditional foods of a touristic region of Sonora State, that can be part of tourism food proposal, since the consumer preferences knowledge. To that aim, a conducted survey was applied to 262 potential tourists, through a choice experiment, where the price, origin and kind of food was the attribute to consider in the choices. A conditional logit model was estimated and found that meat tamales with a low price and from Ures are the most preferred between the consumers.

\section{INTRODUCCIÓN}

La alimentación siempre ha formado parte de los viajes por necesidad biológica, pero fue en la década de 1980 cuando comenzó el desarrollo del viaje con fines gastronómicos exclusivamente. En 1986 la Asociación de Expertos Científicos en Turismo (AIEST) determinó la relación axiomática entre turismo y gastronomía, al encontrar que la comida que se sirve es uno más de los atractivos de un destino turístico junto con los aspectos culturales y naturales (Shlüter, \& Thiel Ellul, 2008). A partir de entonces ha quedado claro que la comida es una importante atracción turística que mejora la experiencia del visitante e incluso para algunos de ellos resulta fundamental.

Para alimentarse, algunos turistas lo hacen durante sus travesías o en su estancia, otros lo consideran un aliciente para iniciar un viaje (Henderson, 2009; Quan, \& Wang, 2004; Tikkanen, 
IIVESTIGACIÓn Y CIERCIA DE LA UNIVERSIDAD AUTÓNOMA DE RGUASCALIERTES
2007). En este sentido, se observa cómo hoy día la oferta alimentaria de comidas regionales/nacionales - también denominadas étnicas desde el punto de vista del consumidor externo a la cultura- están creciendo hasta traspasar el consumo solamente por elementos turísticos para convertirse incluso en una búsqueda de alimentos exóticos, o bien, una manera de acercarse a sitios que los consumidores desearían conocer (Batres-Márquez, Jensen, \& Brester, 2003; Camarena Gómez, \& Sanjuan López, 2008; DataMonitor, 2005; Sandoval Godoy, \& Camarena Gómez, 2011, 2015).

De acuerdo con Flavián y Fandos (2010), las nuevas motivaciones turísticas de tipo cultural (vinculadas por ejemplo a patrimonio arquitectónico - festividades regionales), o bien del tipo hedónico (relacionadas con la creación artística y gastronomía), son punta de lanza de una oferta turística que cada vez seduce a más visitantes. La gastronomía se está convirtiendo en una motivación de viaje cada vez más importante. En España se estiman alrededor de 5 millones y medio que visitan el país por este motivo (Ansón, 2010).

Experiencias en distintas zonas del mundo han demostrado que los alimentos son, sin lugar a dudas, un producto turístico que se vincula con lo local y tradicional (Álvarez, \& Sammartino, 2009). En países europeos las experiencias del turismo se vinculan estrechamente con la alimentación, y ésta a su vez con alimentos que cuentan con apelativos de calidad (Everett, \& Aitchison, 2008; Sims, 2009; Tregear, Arfini, Belletti, \& Marescotti, 2007). En otras zonas, el turismo alimentario se encuentra en pleno proceso de crecimiento, tal como sucede en Nueva Zelanda y Australia, donde las rutas del vino se desarrollan con gran pujanza (Alonso, 2009; Bruwer, \& Alant, 2009; Duarte-Alonso, \& Northcote, 2009).

Han surgido propuestas de turismo alimentario, de las que son parte las rutas alimentarias y gastronómicas. La experiencia en trayectos alimentarios es muy amplia; no obstante, en América Latina comenzó a desarrollarse a partir del año 2000 a través de una propuesta planteada en Argentina como un Programa Nacional de Turismo Rural (Barrera, \& Bringas-Alvarado, 2009; Schlüter, \& Thiel Ellul, 2008). A partir de la experiencia argentina, en otros países de Latinoamérica se comenzó a trabajar en este tipo de iniciativas; algunas de ellas ya se encuentran en marcha, como la Ruta del tequila en México y la del café en Colombia, mientras que en otros casos, como el de la Ruta de la sal en México y la del desierto en Chile se encuentran como propuestas o en desarrollo (Ascanio, 2009; Barrera, \& Bringas-Alvarado, 2009). Otros casos, como los viñedos de California en Estados Unidos y la vinculación gastronómica en la región del Niágara (Sabores del Niágara, Festival de la uva y vino) en Canadá, se muestran como ejemplos de turismo alimentario (Jayawardena, Patterson, \& Brain, 2008; Stewart, Bramble, \& Ziraldo, 2008; Warner, 2007).

Resulta evidente que el turismo relacionado con los alimentos ha tomado mayor fuerza en los últimos años, pero el desarrollo no siempre es igual en todas las regiones, incluso al interior de un país. En este sentido, se observa cómo la cocina tradicional mexicana ha sido reconocida por la UNESCO como patrimonio inmaterial de la humanidad, distintivo que implica una afirmación a las características de originalidad y tradición que integran la cocina nacional, pero al mismo tiempo una oportunidad de potenciar el turismo gastronómico (UNESCO, 2009).

En el noroeste del país las experiencias de turismo alimentario son escasas, más en el estado de Sonora. Si bien la actividad turística tiene una importante influencia social y económica, lo cierto es que no todas las regiones del estado se han visto favorecidas, en especial las ubicadas en zonas rurales. Por ejemplo, en 2015 se registró una afluencia de 2, 407, 107 turistas e ingresos de 4, 100 millones de pesos (Acuña, 2015; SECTUR, 2016). Estas cifras permiten dimensionar el impacto que genera este aspecto en el estado, especialmente en los sitios importantes receptores de turistas, como destinos de playa y urbanos, principalmente (COFETUR, 2008). Por ello han surgido diversas iniciativas, tanto de organismos oficiales, como privados y académicos, para impulsar el turismo en las zonas rurales. Se han conformado algunos circuitos en el interior como Río Sonora, las Misiones, Yécora, Sierra Alta y Sierra Mar, incluidos en algunos de ellos los denominados pueblos mágicos (Sonora Turismo, 2010). Aunque estas opciones se han convertido en propuestas interesantes para estimular este rubro en algunos pueblos de la entidad (Salido Araiza et al., 2009), es conveniente destacar que tales trayectos no se vinculan con otros componentes económicos esenciales de los cuales depende una amplia parte de la población que habita las localidades que integran las rutas.

En correspondencia con esa necesidad de generar mayores oportunidades de desarrollo económico para la población rural y retomando ese 
nexo entre alimentación, desarrollo rural y turismo, surgió la propuesta de esta investigación, donde el objetivo principal consistió en identificar los alimentos tradicionales que pueden integrarse dentro del turismo alimentario a partir del conocimiento de las preferencias de los consumidores. Se utilizó como metodología de investigación el experimento de elección, y se estima un modelo logit condicional a partir del cual se determinan los alimentos que los consumidores prefieren e identifican con una región, así como la disposición a pagar por ellos.

No hay que perder de vista que de acuerdo con la ley de la oferta y la demanda, un menor precio conlleva una mayor concurrencia en el mercado, y que en el caso del origen

\footnotetext{
el arraigo y la costumbre son factores que resultan favorecedores para la continuidad de la demanda de alimentos tradicionales. Sin embargo, cambios en las preferencias y necesidades de la población no deben perderse de vista, por lo que se vuelve necesario generar nuevos esquemas, formatos de presentación y comercialización que resulten más atractivos para los distintos segmentos de consumidores. De momento, los alimentos típicos sonorenses siguen contando con el beneplácito de la población. (Sandoval Godoy, \& Camarena Gómez, 2012, p. 71)
}

La hipótesis de investigación se orienta a considerar que las preferencias de los compradores favorecerán los productos con menor precio y que mantienen la tradición, además de tener un origen conocido por ellos.

Cabe destacar que de las opciones y regiones donde se implementan actividades de turismo rural en el estado, aquí se trabaja en la ruta del río Sonora, específicamente el municipio de Ures. La elección obedece a sus indicadores, ya que se muestra un crecimiento constante y sostenido, además cuenta con los factores de pasado histórico, cultura e infraestructura necesarios para consolidarse como trayecto turístico de gran potencial.

\section{Caracterización de la zona de estudio}

El turismo rural en Sonora se conforma mayoritariamente por viajeros regionales y nacionales, con una estancia media de 3.3 d (COFETUR, 2008). Se presenta una alta estacionalidad de la demanda, ya que el mayor flujo se concentra en fechas vacacionales de verano y semana santa, con menor incidencia en navidad, puentes o días festivos. De las zonas rurales, la Ruta del río Sonora concentra $25 \%$ de los turistas (COFETUR, 2007). Se integra por nueve pueblos: Ures, Baviácora, Aconchi, San Felipe de Jesús, Huépac, Banámichi, Arizpe, Bacoahi y Cananea. Estos municipios comprenden una superficie total de $13,418 \mathrm{~km}^{2}, 7.2 \%$ de la superficie del territorio estatal. Los municipios con mayor concentración territorial son Cananea, Arizpe y Ures, que juntos abarcan una superficie de $9,556 \mathrm{~km}^{2}$. Si se toma como punto de partida Hermosillo, se llega a través de un camino paralelo al río Sonora, con una distancia de $78 \mathrm{~km}$ en el punto más cercano (Ures) y de $295 \mathrm{~km}$ al más distante (Cananea) (Ruta río Sonora, 2017).

Del total de visitantes de la ruta del río Sonora $88 \%$ son nacionales, de los cuales $60.1 \%$ proceden de la ciudad de Hermosillo. En cuanto a la recepción de turistas por municipio, la mayor concentración se ubica en Ures (58.4\%), después Aconchi (41.6\%) y Baviácora y Banámichi (35.5\% cada uno). En general, Ures se posiciona como el municipio con mayor captación de visitas de este trayecto (COFETUR, 2008). Los turistas que visitan este municipio proceden en $90 \%$ de Hermosillo (Salido Araiza et al., 2009). Asimismo, debido a la cercanía con este entorno urbano ( $65 \mathrm{~km}$ al este), existe una gran afluencia de visitantes durante los fines de semana para degustar los platillos típicos de la región, entre los cuales se encuentran los tamales de carne o de elote, la carne machaca, las coyotas (tortillas de harina rellena de piloncillo cocinada en horno de leña y utilizadas como postre), los jamoncillos (elaborados con leche y azúcar en una pasta que se mezcla con nuez), la carne con chile, el caldo de queso, el pozole de trigo, entre otras (Camou, 2012). Entre las opciones de platillos sonorenses que se comercializan en esta región se busca identificar los preferidos, así como los emblemáticos.

MATERIALES Y MÉTODOS

\section{La muestra}

Se desarrolló una investigación exploratoria con fuentes de información primarias basadas en la elaboración de encuestas y de entrevistas en profundidad. Las entrevistas personales se hicieron a dueños y encargados de restaurantes de Ures y San Pedro el Saucito durante octubre de 2012. El objetivo fue identificar los alimentos con identidad tradicional y sus características de comercialización, se visitaron 34 establecimientos y en dos se negaron a participar. La encuesta a potenciales turistas se elaboró a partir de la información recabada con las aplicadas a los empresarios de restaurantes de Ures y San Pe- 
IIVESTIGACIÓn Y CIERCIA DE LA UNIVERSIDAD AUTÓNOMA DE RGUASCALIERTES dro, así como de entrevistas a los consumidores, con guion semiestructurado de 10 preguntas, referentes a los alimentos típicos de la ruta del río Sonora, preferencias hacia ellos y actitudes.

Se realizaron 13 entrevistas a compradores de la ciudad de Hermosillo, con promedio de 15 a 20 minutos de duración cada una. El cuestionario se aplicó a 262 consumidores, $90 \%$ procedía de Hermosillo y $10 \%$ personas que habitan fuera del municipio. Se utilizó esta distribución porque se asemeja a las características de la afluencia turística de la ruta del río Sonora. Las personas que participaron en las encuestas se seleccionaron totalmente al azar, en sitios públicos de esparcimiento como parques, así como restaurantes de destinos rurales (en la zona del río Sonora), en las inmediaciones de establecimientos de compra, entre otros. Ningún participante percibió compensación económica.

\section{Diseño del experimento de elección}

Una inspección de la oferta gastronómica en Ures y San Pedro el Saucito, tanto mediante visitas a restaurantes como a otros establecimientos de comida, así como entrevistas a consumidores, permitieron definir los niveles específicos de los atributos seleccionados para el experimento que se presentan en la tabla 1. Para mantener el experimento dentro de parámetros manejables, de las distintas cocinas típicas se designaron las tres más vendidas y mencionadas por los consumidores: tamales de carne, tamales de elote y el taco de carne con chile.

En cuanto a su preparación, tanto los tamales de elote como los de carne se hacen con una pasta de harina de maíz como base, colocada en una hoja de la mazorca de la misma planta de maíz y se cocinan en una cacerola al vapor. Los de elote únicamente contienen la pasta de maíz y queso, los segundos carne deshebrada condimentada con algo de chile (picante). Los tacos de carne con chile (también conocidos regionalmente como burros) se elaboran envolviendo en una tortilla de trigo (preparación de harina de trigo sin levadura) carne de res deshebrada con chile. Los tres alimentos son comunes a las tres alternativas de origen (Ures, entorno urbano, otro entorno rural), mientras que el rango de precios se define para cada alternativa de consumo.

El número total de combinaciones o productos que se pueden obtener con $A$ atributos y $L$ niveles (dado que todos los atributos tienen el mismo número de niveles) es LA, lo que se traduce en 27 (3(3)) com-
Tabla 1

Niveles y atributos de los productos hipotéticos del experimento de elección

\begin{tabular}{|c|c|c|}
\hline \multicolumn{3}{|c|}{ Atributos } \\
\hline Comida típica & Origen* & Precio ** \\
\hline Tamales de elote & & \\
\hline Tamales de carne & $\begin{array}{l}\text { Entorno urbano } \\
\text { Otro entorno }\end{array}$ & $\begin{array}{l}18 \text { pesos } \\
22 \text { pesos } \\
26 \text { pesos }\end{array}$ \\
\hline Carne con chile & & \\
\hline
\end{tabular}

Nota: * Otro entorno rural puede ser cualquier otro pueblo excepto Ures. ** Los precios son por una orden de dos tamales o por medio taco de carne con chile.

Elaboración propia.

binaciones. Con el objeto de hacer el experimento más manejable se ha distribuido el conjunto total de tarjetas en subgrupos, de manera que cada entrevistado tan sólo valora uno de los bloques con un número más reducido de tarjetas. Para conseguir que la distribución de tarjetas en bloques sea totalmente aleatoria e incorrelacionada con los atributos, el bloque se convierte en un atributo adicional a la hora de realizar el diseño (Henser, Rose, \& Greene, 2005).

El diseño ortogonal en combinación con la distribución por bloques ha sido realizado mediante la función integrada del paquete Ngene. De esta forma, el experimento final cuenta con 27 productos (o combinaciones alternativas de nivel y atributo), distribuidas en 3 bloques de 9 tarjetas cada uno. En cada tarjeta, además de las dos alternativas etiquetadas por la situación de consumo, se incluye la al-

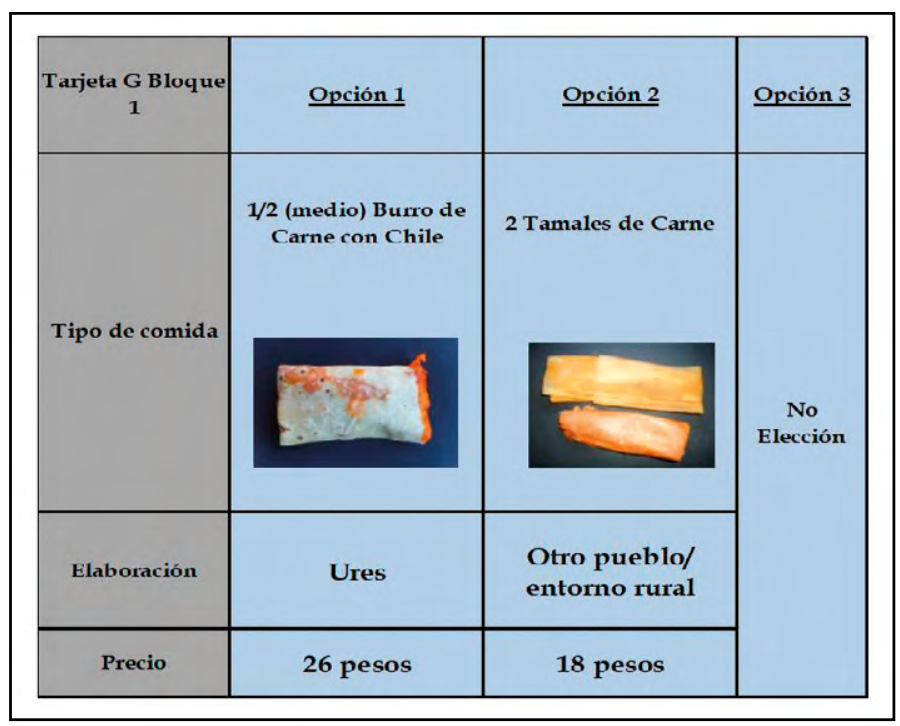

Figura 1. Diseño de tarjetas mostradas al consumidor turístico. Elaboración propia. 
Tabla 2

Descripción de las variables explicativas del modelo logit

\begin{tabular}{ll}
\hline \multicolumn{1}{c}{ Denominación } & \multicolumn{1}{c}{ Descripción } \\
\hline Precio & $\begin{array}{l}\text { Variable continua } \\
\text { alternativa es elaborada en Ures; y } 0 \text { si la alternativa es elaborada en otro rural } .\end{array}$ \\
\hline Tipo de comida & $\begin{array}{l}\text { alternativa posee la característica de tamal de carne; y } 0 \text { si la alternativa posee la } \\
\text { característica de tamal de elote. }\end{array}$ \\
\hline$\alpha_{i}$ & Constante específica para la alternativa i (i= A, B) \\
\hline
\end{tabular}

Nota: Elaboración propia.

ternativa de No elección. En la figura 1 se puede observar un ejemplo de las tarjetas que se presentaron a los consumidores.

\section{Especificación del modelo}

Para modelizar las preferencias del potencial turista de la ruta del río Sonora, se ha estimado un modelo logit condicional. La literatura reserva la denominación condicional para los modelos de elección discreta en que las variables explicativas son los atributos que caracterizan las alternativas de elección. En este caso, tales variables son: origen, precio y tipo de comida (tabla 2).

Las variables correspondientes a las características de las alternativas de elección se introducen en el modelo como efectos diferenciales sobre un nivel de referencia (effects codes) en lugar de como variables dummy (Louviere, Henser, \& Swait, 2000). Para exponer los resultados se muestra el modelo más restrictivo, o logit condicional. La utilidad que el individuo $n$ obtiene de la alternativa $i$ ( $i=$ opción $A$ u opción B) se define como:

$U_{i}^{n}=\alpha_{I}+\beta_{p r, i} * \operatorname{Pr}_{i}+\beta_{\text {origen }, i} *$ Origen $_{i}+\beta_{\text {comida }, i} *$ comida $_{i}+\varepsilon_{\text {in }}$

\section{RESULTADOS}

En la tabla 3 se muestran los resultados de la estimación del modelo logit condicional. El signo de los parámetros estimados proporciona información sobre el efecto que la presencia de la característica (en variables categóricas) 0 el aumento (disminución) de una variable continua tiene sobre la probabilidad de elegir un producto que incluya tales atributos. En primer lugar, las variables explicativas son conjuntamente significativas. Al mismo tiempo se advierte que el logaritmo de la función de verosimilitud [LL $(\theta)$ ], denota que, en media, la utilidad de cualquiera de las dos opciones presentadas a los entrevistados es superior a la que se deriva de la opción de no elección.

Si se recorren los parámetros estimados se puede concluir que el precio tiene un efecto negativo. A medida que el precio de las comidas típicas disminuye la utilidad para los consumidores aumenta, por lo que si el precio de las comidas es más bajo, la probabilidad de que los consumidores las compren es mayor, lo cual no es extraño ya que

Tabla 3

Parámetros estimados en el modelo logit condicional básico

\begin{tabular}{|c|c|c|}
\hline Variable & Coeficiente & Prob. \\
\hline a & $0.171^{* * *}$ & 0.004 \\
\hline$a_{B}$ & $0.011^{* * *}$ & 0.000 \\
\hline$\beta_{\mathrm{Pr}}$ & $-0.254^{* * *}$ & 0.000 \\
\hline$\beta_{\text {carne }}$ & $1.111^{* * *}$ & 0.000 \\
\hline$\beta_{\text {elote }}$ & $-0.297^{* * *}$ & 0.002 \\
\hline$\beta_{\text {ures }}$ & $0.477^{* * *}$ & 0.000 \\
\hline$\beta_{\text {urban }}$ & $0.229^{* * *}$ & 0.013 \\
\hline $\begin{array}{l}\text { Num. } \\
\text { Obs: }\end{array}$ & 2304 & \\
\hline LL(0): & $-1576,994$ & \\
\hline $\operatorname{LL}(\theta):$ & $-1291,71$ & \\
\hline LLR: $\chi_{2}^{2}:$ & $390,47(0,000)$ & \\
\hline
\end{tabular}

Nota: LL(0): Logaritmo de la función de verosimilitud evaluada en un modelo donde se incluyen sólo las constantes; LL(e):Logaritmo de la función de verosimilitud evaluada en el modelo con todas las variables explicativas; LLR: Ratio de verosimilitud, entre el modelo donde se incluye sólo las constantes y el modelo básico LLR = -2[LL (0)-LL ( $\theta)]$; LLR sigue una distribución $X^{2}$ con grados de libertad igual al número de coeficientes cuya significatividad conjunta se contrasta. El valor de probabilidad entre paréntesis. "*" Indica significatividad al $1 \%$.

Elaboración propia. 
IIVESTIGACIÓn Y CIERCIA DE LA UNIVERSIDAD AUTÓNOMA DE RGUASCALIERTES la mayoría de los alimentos se caracterizan por ser productos de demanda normal y elástica, donde las variaciones en el precio afectan sensiblemente a la demanda. En cuanto al tipo de comida, se observa que la probabilidad de comprar tamales de carne es mayor que la de comprar tamales de elote $(-0.297)$ e incluso un taco de carne con chile $(-0.814)$. El coeficiente del nivel excluido como variable se puede obtener cambiando el signo de la suma de los coeficientes de los niveles presentes (p. ej. $\beta$ taco $=-\beta$ carne $+\beta$ elote $)$.

En lo que respecta al origen, tanto la comida que procede de Ures (0.477) como del entorno urbano (0.229) tienen mayor probabilidad de compra que una de otro entorno rural $(-0.248)$, y es sensiblemente mayor la probabilidad de elección si alguno de los alimentos procede específicamente de Ures. Es decir, el nombre de Ures ya tiene una preferencia entre los consumidores, aunque la realidad de los mercados muestra que el atributo origen es escasamente promocionado.

A partir de la estimación del modelo logit es posible conocer la disposición marginal a pagar por un nivel específico de un atributo, el cual se obtiene como el cociente entre el parámetro de dicho nivel/ atributo y el coeficiente del precio, cambiado de signo (Train, 2003):

$$
\begin{array}{cl}
\text { donde: } & \mathrm{DMP}_{\mathrm{i}}=-\frac{\beta_{\mathrm{i}}}{\beta_{\mathrm{PR}}} \\
\mathrm{DMP}_{\mathrm{i}}=\begin{array}{l}
\text { Disposición Marginal a Pagar por el } \\
\text { nivel/atributo } \mathrm{i}
\end{array} \\
\beta_{\mathrm{i}}=\quad \begin{array}{l}
\text { Coeficiente del nivel/atributo } \mathrm{i} \\
\beta_{\mathrm{PR}}=
\end{array}
\end{array}
$$

En este caso, los resultados indican que por tipo de comida los consumidores estarían dispuestos a pagar un sobreprecio de hasta 4.37 pesos por un tamal de carne, pero que en el caso de los tamales de elote y tacos de carne con chile es necesario realizar un descuento de 1.16 pesos en el caso del primero y de 3.20 en el segundo. En el caso del origen consideran que es factible pagar un sobreprecio de 1.87 pesos cuando el producto procede de Ures, de 0.90 si es elaborado en un entorno urbano, pero en el caso de tener origen en otro entorno rural que no sea Ures es necesario compensarles con un descuento de 0.97 pesos (tabla 4).

\section{DISCUSIÓN}

El experimento de elección es utilizado en diversas áreas del conocimiento, en el ámbito del turismo y sus distintas vertientes cada vez son más comunes las investigaciones que hacen uso de esta metodología (Chaminuka, Groeneveld, Selomane, \& van lerland, 2012; Lyu, 2017; Oppewal, Huybers, \& Crouch, 2015; Román, \& Martín, 2016). Los resultados muestran que en cuanto al precio existe una relación inversa, ya que a medida que aumenta la utilidad de los consumidores disminuye; estos resultados son congruentes con la teoría económica y con lo encontrado en otras investigaciones alimentarias y de turismo (Alemu, Olsen, Vedel, Pambo, \& Owino, 2017; Aoki, Akai, \& Ujiie, 2017; Asioli, Almli, \& Naes, 2016; Lyu, 2017).

En cuanto al atributo origen, se ha visto que existe preferencia hacia las comidas que proceden de Ures, esta predilección puede enmarcarse en la tradición que existe en este municipio en la venta de alimentos, además de tratarse de comidas familiares para el consumidor. Estos resultados son coincidentes con los obtenidos por Camarena Gómez y Sanjuan López (2010) donde se demostró que los consumidores tienden a preferir los productos (harina de maíz) procedentes de su país de origen. En la misma línea, los resultados de Asioli et al. (2016) muestran que el origen nacional o regional prima sobre el externo, incluso en algunos casos sobre atributos intrínsecos del producto (Aoki et al., 2017;

Tabla 4

Disposición marginal al pago

\begin{tabular}{lccc}
\hline \multicolumn{1}{c}{ Atributo } & Disposición al pago/ \$ & \multicolumn{1}{c}{ Atributo } & Disposición al pago/ \$ \\
\hline Tamal de carne & 4.37 & Origen Ures & 1.87 \\
\hline Tamal de elote & -1.16 & Origen urbano & 0.90 \\
\hline Taco de carne con chile & -3.24 & Origen otro entorno rural & -0.97 \\
\hline
\end{tabular}

Nota: Elaboración propia. 


\section{InVESTIGACIÓn Y CIERCIA DE LA UחIVERSIDAD AUTÓnOMH DE RGUASCALIETTES}

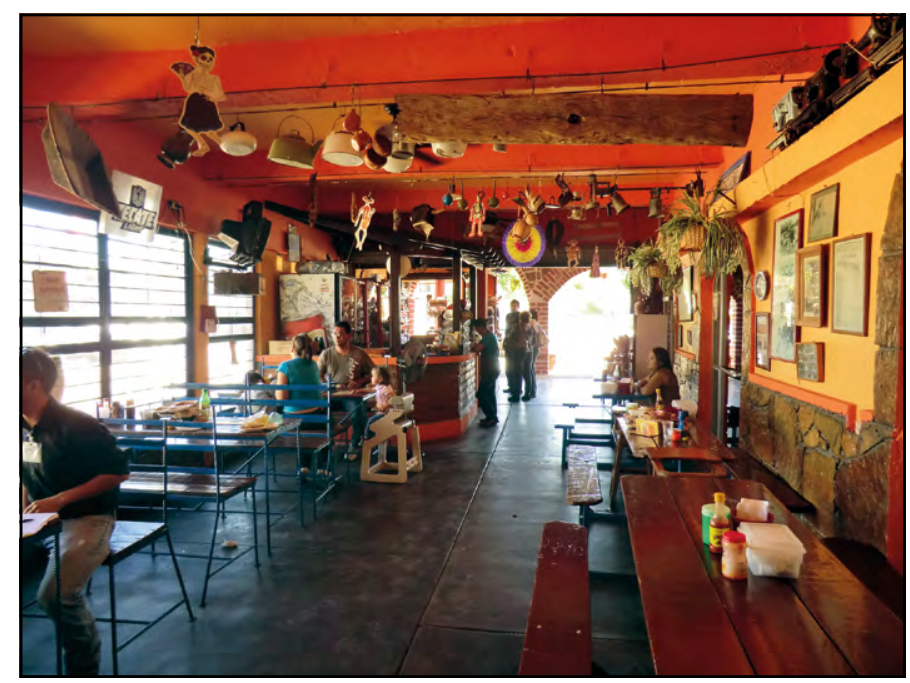

Figura 2. Se estudian los alimentos tradicionales que pueden integrarse dentro del turismo alimentario a partir de las preferencias de los consumidores.

Fotografía proporcionada por la autora.

Dekhili, Sirieix, \& Cohen, 2011; Kim, 2008; Schnettler, Ruiz, Sepúlveda, \& Sepúlveda, 2008).

En lo relativo al tipo de comida, los resultados se orientan hacia la preferencia del tamal de carne, producto con arraigo en la cultura gastronómica mexicana por tener como materia prima el maíz (Ramírez-Vidal, 2005). Lo cierto es que diversos estudios han encontrado una preferencia de los consumidores sonorenses hacia los tamales que incluyen carne como ingrediente complementario (Sandoval Godoy, \& Camarena Gómez, 2015; Sandoval Godoy, Domínguez lbáñez, \& Cabrera Murrieta, 2010). En este sentido, los hallazgos se relacionan con los encontrados por Alemu et al. (2017) y Balogh, Békési, Gorton, Popp y Lengyel (2016), donde la costumbre, la tradición y conocimiento pueden llegar a favorecer el consumo de un producto.

\section{CONCLUSIONES}

El turismo alimentario es una alternativa económica que se ha desarrollado con éxito en varios países del mundo. La tradición, el origen y la autenticidad son elementos que permiten posicionar los platillos en el gusto de los consumidores y potenciarlos como parte de la dinámica del turista. La investigación que aquí se presentó es un primer esbozo sobre opciones que se pueden potenciar si se toman en cuenta la demanda del mercado, las ventajas competitivas de las regiones rurales y el movimiento que se desarrolla en torno al turismo (figura 2).
No obstante, pensar en una estrategia de turismo alimentario desvinculada de las necesidades y demandas actuales del mercado, resulta arriesgado y con limitaciones. De ahí que el objetivo haya sido conocer las preferencias que manifiestan los potenciales consumidores turistas sobre alimentos tradicionales de Ures, mediante un experimento de elección. Los resultados del mismo muestran que los consumidores prefieren una comida de tamales frente a los tacos de carne con chile, pero que el tamal de carne es el preferido sobre el de elote. Asimismo, el origen Ures es preferido sobre otros, lo que deja entrever la fama o conocimiento que los consumidores tienen sobre los alimentos típicos de la ruta del río Sonora. Por ello, el producto preferido es el tamal de carne, a un precio bajo con origen de Ures, que puede llegar a tener un incremento en el precio si se destaca su origen y proceso tradicional de elaboración. Estos datos permiten comprobar la hipótesis de preferencia hacia el origen y conocimiento del producto, así como su relación inversa con el precio.

Esta investigación constituye el punto de partida para el análisis de las estrategias de desarrollo regional basadas en el turismo rural. El consumidor analizado como turista potencial se vuelve eje fundamental, por lo que su opinión, percepción y conocimiento de las opciones de turismo rural se vuelven la punta de lanza para la implementación de estrategias.

Desde el punto de vista de las implicaciones empresariales, el desarrollo y mejora de los servicios, así como la atención al cliente, se vuelven medulares en el éxito de un establecimiento. El origen, autenticidad y nostalgia son elementos que pueden desarrollarse más en los productos que se ofrecen, con el fin de atraer a un mayor número de visitantes. En el caso de Ures las opciones de turismo de fines de semana son modalidades en las que queda mucho por hacer, pero no deben de perderse de vista, ya que actualmente son las que realiza y demanda el consumidor.

El tamal de carne es un alimento que se posiciona en la mente de los consumidores como tradicional y típico de Ures, por lo que puede considerarse el punto de partida para desarrollar un turismo de trayectos alimentarios, el cual puede complementarse con otros productos que se comercializan en la región como las cañas de azúcar, el piloncillo, los ponteduros de palomitas de maíz, jamoncillos, entre otros. 
IIVESTIGACIÓn Y CIERCIA DE LA UNIVERSIDAD AUTÓNOMA
Por último, la investigación se llevó a cabo en una zona con características muy específicas, por lo que su extrapolación hacia otros entornos debe realizarse con cautela. Asimismo, su análisis puede am- pliarse, con una muestra más nutrida, incluir otras regiones, distintas épocas del año y productos como artesanías, entre otros.

REFERENCIAS

- Acuña, D. (2015). Estiman que el turismo deje derrama económica en Sonora por 4 mil 100 millones de pesos. El imparcial, sección Sonora, 25 de junio de 2015. Recuperado en diciembre de 2016, de http://www.elimparcial.com/ EdicionEnlinea/Notas/Sonora/25072015/992590-Estiman-queel-turismo-deje-derrama-economica-en-Sonora-por-4-mil100-millones-de-pesos.html

- Alemu, M. H., Olsen, S. B., Vedel, S. E., Pambo, K. D., \& Owino, V. O. (2017). Combining product attributes with recommendation and shopping location attributes to assess consumer preferences for insect-based food products. Food Quality and Preference, 55, 45-57.

- Alonso, A. D. (2009). Are travelers interested in wine tourism in New Zealand? International Journal of Culture, Tourism and Hospitality Research, 3(1), 13-24.

- Álvarez, M., \& Sammartino, G. (2009). Empanadas, tamales y carpaccio de llama: Patrimonio alimentario y turismo en la Quebrada de Humahuaca-Argentina. Estudios y Perspectivas en Turismo, 18(2), 161-175.

- Ansón, R. (2010). Conferencia Inaugural. En I Congreso Europeo del Turismo y la Gastronomía. 24 y 25 de mayo, Madrid, España: Secretaría del Estado de Turismo.

- Aoki, K., Akai, K., \& Ujiie, K. (2017). A choice experiment to compare preferences for rice in Thailand and Japan: The impact of origin, sustainability, and taste. Food Quality and Preference, 56, 274-284.

- Ascanio, A. (2009). Rutas gastronómicas chilenas: Una aproximación al tema. PASOS Revista de Turismo y Patrimonio Cultural, 7(2), 321-325.

- Asioli, D., Almli, V. L., \& Naes, T. (2016). Comparison of two different strategies for investigating individual differences among consumers in choice experiments. A case study based on preferences for iced coffee in Norway. Food Quality and Preference, 54, 79-89.

- Balogh, P., Békési, D., Gorton, M., Popp, J., \& Lengyel, P. (2016). Consumer willingness to pay for traditional food products. Food Policy, 61, 176-184.

- Barrera, E., \& Bringas-Alvarado, O. (2009). Rutas alimentarias: Una estrategia de negocios inclusivos que vincula las políticas agrarias y turísticas. Études Caribéenes, 13-14. Recuperado en agosto de 2012, de http://etudescaribeennes.revues.org/3828
- Batres-Marquez, S. P., Jensen, H., \& Brester, G. (2003). Salvadoran consumption of ethnic foods in the United States. Journal of Food Distribution Research, 34(2), 1-16.

- Bruwer, J., \& Alant, K. (2009). The hedonic nature of wine tourism consumption: an experiental view. International Journal of Wine Business Research, 21 (3), 235-257.

- Camarena Gómez, D. M., \& Sanjuan López, A. I. (2008). El mercado de comida étnica en España: el caso de la comida mexicana. Estudios Sociales, 16(31), 7-37.

- (2010). Preferencias hacia el origen de un alimento étnico y la influencia de variables psicográficas. Economía Agraria y de Recursos Naturales, 10(1), 71-99.

- Camou, E. (2012). Raíces de nuestra identidad: Historia, alimentación y cultura. En M. C. Hernández, \& J. M. Meléndez, Alimentación contemporánea: un paradigma en crisis y respuestas alternativas. México: CIAD, A. C.-AM Editores.

- Chaminuka, P., Groeneveld, R. A., Selomane, A. O., \& van lerland, E. C. (2012). Tourist preferences for ecotourism in rural communities adjacent to Kruger National Park: A choice experiment approach. Tourism Management, 33(1), 168-176.

- Comisión de Fomento al Turismo. (2007). Plan rector para el desarrollo turístico sustentable de la ruta del río Sonora. Reporte técnico. Sonora, México: CIAD-COFETUR Sonora. agosto de 2012, de http://institucional.sonoraturismo.gob.mx/ estadisticas.htm

- DataMonitor (2005). Insights into tomorrow's ethnic food \& drink consumers (pp. 84). Reference Code: DMCM2363.

- Dekhili, S., Sirieix, L., \& Cohen, E. (2011). How consumers choose olive oil: The importance of origin cues. Food Quality and Preference, 22(8), 757-762.

- Duarte-Alonso, A., \& Northcote, J. (2009). Wine, history, landscape: Origin branding in Western Australia. British Food Journal, 111 (11), 1248-1259.

- Everett, S., \& Aitchison, C. (2008). The role of food tourism in sustaining regional identity: A case study of Cornwall, South West England. Journal of Sustainable Tourism, 16(2), 150-167.

- Flavián, C. y Fandos, C. (2011). Turismo gastronómico. Estrategias de marketing y experiencias de éxito. Murcia, España: Prensas Universitarias de Zaragoza. 


\section{IIVESTIGAGIÓn Y CUERGIA DE LA UחIVERSIDAD AUTÓnOMTH DE RGUASCHLIETTES}

- Henderson, J. (2009). Food tourism reviewed. British Food Journal, 111 (4), 317-326.

- Henser, D., Rose, J., \& Greene, W. (2005). Applied Choice Analysis. A primer. New York, NY: Cambridge University Press.

- Instituto Nacional de Estadística y Geografía. (2011). México en Cifras. Recuperado en 2013, de http://www.inegi.org.mx/

- Jayawardena, C., Patterson, D., \& Brain, R. (2008). Sustainable tourism development in Niagara: Discussions, theories, projects and insights. International Journal of Contemporary Hospitality Management, 20(3), 258-277.

- Kim, R. (2008). Japanese consumers' use of extrinsic and intrinsic cues to mitigate risky food choices. International Journal of Consumer Studies, 32 (1), 49-58.

- Louviere, J. J., Henser, D. A., \& Swait, J. D. (2000). Stated choice methods: analysis and application. New York: Cambridge University Press.

- Lyu, S. O. (2017). Which accessible travel products are people with disabilities willing to pay more? A choice experiment. Tourism Management, 59, 404-412.

- Oppewal, H., Huybers, T., \& Crouch, G. I. (2015). Tourist destination and experience choice: A choice experimental analysis of decision sequence effects. Tourism Management, $48,467-476$.

- Quan, S., \& Wang, N. (2004). Towards a structural model of the tourist experience: an illustration from food experiences in tourism. Tourism Management, 25(3), 297-305.

- Ramírez Vidal, L. A. (2005). Reseña de ¡Vivan los tamales! La comida y la construcción de la identidad mexicana. Alteridades, 15(29), 143-146.

- Román, C., \& Martín, J. C. (2016). Hotel attributes: Asymmetries in guest payments and gains-A stated preference approach. Tourism Management, 52, 488-497.

- Ruta río Sonora (2017). Recuperado en enero de 2017, de http://www.arduinna.com.mx/es/ruta_turistica_rio_sonora. html

- Salido Araiza, P. L., Bañuelos Flores, N., Romero Escalante, D. M., Romo Paz, E. L., Ochoa Manrique, A. I., Rodica Caracuda, A., \& Olivares Cervantes, J. (2009). El patrimonio natural y cultural como base para estrategias de turismo sustentable en la Sonora Rural. Revista de Estudios Sociales, 17(Número Especial), 81-103.

- Sandoval-Godoy, S. A., \& Camarena-Gómez, D. M. (2011). Comportamiento alimentario y perfil de consumo de los sonorenses: el caso de las comidas internacionales. Revista Región y Sociedad, 23(50), 185-213.
(2012). Consumo de alimentos de la población sonorense: tradición versus internacionalización. Estudios Sociales, 20(2), 53-72. editores-Centro de Investigación en Alimentación y Desarrollo (CIAD).

- Sandoval-Godoy, S. A., Domínguez-lbáñez, S., \& CabreraMurrieta, A. (2010). De golosos y tragones están llenos los panteones: cultura y riesgo alimentario en Sonora. Estudios Sociales, 17, 149-179.

- Schlüter, R. G., \& Thiel Ellul, D. (2008). Gastronomia y turismo en Argentina Polo gastronomic Tomas Jofré. PASOS. Revista de Turismo y Patrimonio Cultural, 6(2), 249-268.

- Schnettler, B., Ruiz, D., Sepúlveda, O., \& Sepúlveda, N. (2008). Importance of the country of origin in food consumption in a developing country. Food Quality and Preference, 19(4), 372382.

- Secretaría de Turismo. (2016). Información turística por entidad federativa. Recuperado en diciembre de 2016, de http:// www.datatur.sectur.gob.mx/ITxEF/ITxEF_SON.aspx

- Sims, R. (2009). Food, place and authenticity: local food and the sustainable tourism experience. Journal of Sustainable Tourism, 17(3), 321-336.

- Sonora Turismo (Comisión de Fomento al Turismo del Estado de Sonora). (2010). Rutas turísticas de Sonora. Recuperado en agosto de 2012, de http://www.sonoraturismo.gob.mx/ destinos-sonora/rutas.html

- Stewart, J. W., Bramble, L., \& Ziraldo, D. (2008). Key challenges in wine and culinary tourism with practical recommendation. International Journal of Contemporary Hospitality Management, 20(3), 303-312.

- Tikkanen, I. (2007). Maslow's hierarchy and food tourism in Finland: Five cases. British Food Journal, 109(9), 721-734.

- Train, K. (2003). Discrete choice methods with simulation. US: Cambridge University Press.

- Tregear, A., Arfini, F., Belletti, G., \& Marescotti, A. (2007). Regional foods and rural development: The role of product qualification. Journal of Rural Studies, 23(1), 12-22.

- United Nations Educational, Scientific and Cultural Organization. (2009). Intangible cultural heritage. Recuperado en enero de 2017, de http://www.unesco.org/culture/ich/ doc/src/01851-ES.pdf

- Warner, K. D. (2007). The quality of sustainability: Agroecological partnership and the geographic branding of California winegrapes. Journal of Rural Studies, 23(2), 142-155. 